\title{
Más allá del sodio: cambios en la dieta y su efecto en hipertensión
}

\author{
María Magdalena Farías ${ }^{1}$, Ada Cuevas ${ }^{1}$, Héctor Ducci ${ }^{2}$
}

Departamentos de Nutrición ${ }^{1}$ y Cardiología ${ }^{2}$, Clínica Las Condes.

Resumen: La hipertensión arterial (HTA) es un importante factor de riesgo cardiovascular y su prevalencia aumenta con la edad, dado el aumento de la expectativa de vida de nuestra población esta condición constituye una prioridad a nivel de salud pública. Tanto para el tratamiento como para la prevención de la HTA son importantes las medidas no farmacológicas como cambios en el estilo de vida y especialmente la alimentación. Además de la restricción de sodio, existen otras medias dietéticas que han demostrado estar asociadas con un mejor control de la HTA en diversos ensayos clínicos, tales como la reducción del peso corporal, la dieta rica en frutas y verduras, el mayor consumo de potasio y magnesio, la vitamina $\mathrm{D}$, los ácidos grasos omega-3, los flavonoides y la disminución de la ingesta de sacarosa, fructosa, cafeína y alcohol. Dado la gran prevalencia de HTA en nuestro país del orden de un $26,9 \%$, es importante dar a conocer medidas dietéticas que nos permitirán un mejor control de la presión en pacientes hipertensos y un retraso de la aparición de esta enfermedad en sujetos sanos, además de su incorporación en campañas de educación nutricional poblacional por parte de las autoridades sanitarias.

Palabras claves: hipertensión arterial, dieta, nutrición, sodio, potasio.

\section{Correspondencia:}

María Magdalena Farías Navarro

Departamento de Nutrición, Clínica Las Condes

Lo Fontecilla 441, Santiago, Chile

mfariasn@clc.cl 


\section{Beyond Sodium: Diet changes and their effect on Hypertension}

Hypertension is an important cardiovascular risk factor and its prevalence increases with age. Given the rising life expectancy in Chile, the prevention of hypertension is a priority for public health policies. Non pharmacological therapies, such as life style and diet changes are especially important. In addition to sodium restriction, several clinical studies have shown that other dietary interventions, such as an increased fruit and vegetable content of diet, higher intake of potassium and magnesium, vitamin $\mathrm{D}$, omega-3 fatty

La hipertensión arterial (HTA) es un importante factor de riesgo cardiovascular (CV), tanto en hombres como en mujeres, y su prevalencia aumenta con la edad. En relación al riesgo $\mathrm{CV}$, éste aumenta en forma lineal y progresiva a partir de una presión arterial (PA) tan baja como $115 \mathrm{mmHg}$ de presión sistólica y $75 \mathrm{mmHg}$ de presión diastólica. ${ }^{1}$ Según el estudio Framingham, una PA desde 130-139/85-89 mmHg se asocia al doble de riesgo relativo de eventos $\mathrm{CV}$, al compararla con una PA menor de 120/80 mmHg. ${ }^{2}$ Por lo tanto, el control de la HTA y la prevención de su aparición con la edad son prioridades a nivel de salud pública.

Las medidas no farmacológicas dirigidas a disminuir los niveles de PA pueden ser ampliamente indicadas a todos aquellos pacientes hipertensos y a aquellos en quienes se desea retardar el desarrollo de HTA. Mientras que para iniciar un tratamiento farmacológico, es importante considerar no sólo el grado de la PA sino que también el riesgo cardiovascular total de un paciente. ${ }^{3}$

Desde una visión preventiva, cobran especial importancia aquellos cambios en el estilo de vida, y en especial en la alimentación, destinados a disminuir la PA. Existe una gran asociación entre la dieta y la PA, siendo la reducción del consumo de sodio el factor más estudiado, ${ }^{4}$ con un efecto dosis-respuesta, especialmente en sujetos sensibles. Así, un meta análisis de 56 estudios randomizados concluyó que al disminuir en $100 \mathrm{mmol} /$ día (equivalentes a 2,3 gr/día) en la excreción urinaria de sodio (la cual se correlaciona con la ingesta), se obtiene una baja de 3,7/0,9 mmHg en la PA sistólica/diastólica. ${ }^{4}$ Sin embargo, el efecto de la alimentación en la evolución de la HTA de acids, flavonoids, decreased intake of sucrose, fructose, caffeine and alcohol, lead to better control of hypertension. Given de high prevalence of hypertension in Chile ( $26.9 \%$ overall) better information about the benefits of diet changes might be very important in order to help prevent and delay the appearance of hypertension. Appropriate public educational policies should be implemented by the sanitary authorities.

Keywords: hypertension, diet, nutrition, sodium, potassium.

un individuo no puede centrarse en un único nutriente ya que la situación es mucho más compleja (Figura 1), por lo que otros factores implicados en la PA merecen atención, especialmente si reconocemos la necesidad de realizar intervenciones educativas poblacionales con el fin de prevenir la aparición de esta enfermedad. En esta revisión se detallan dichos factores y sus mecanismos involucrados en la regulación de la PA.

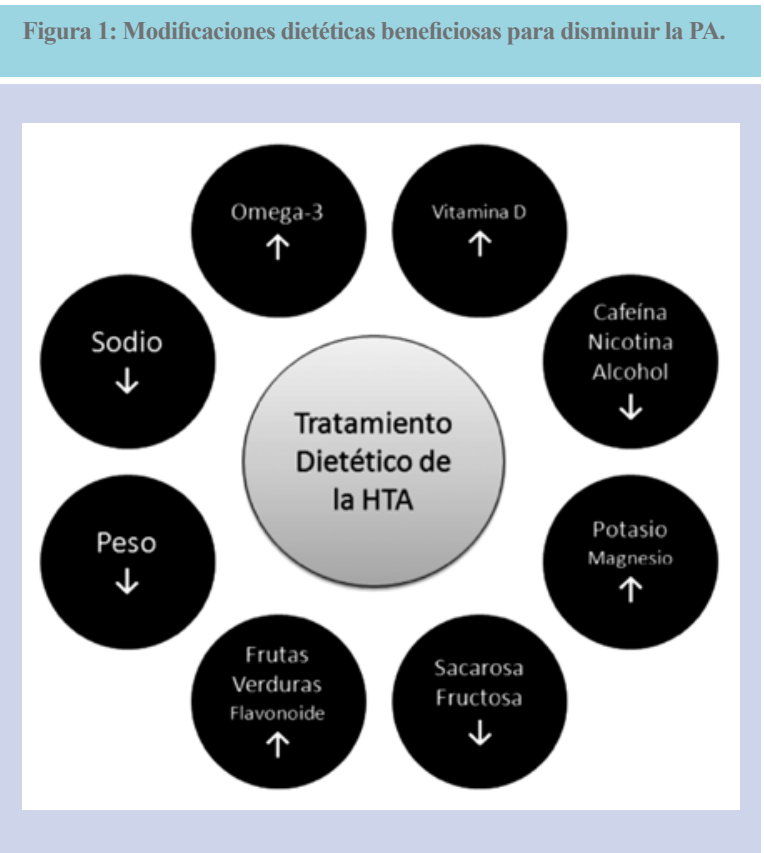




\section{Reducción del peso corporal}

De acuerdo a la Encuesta Nacional de Salud 2010, en Chile el 33,6\% de las mujeres y el 45,3\% de los hombres tienen sobrepeso y un $25,1 \%$ presenta obesidad, definido como un índice de masa corporal (IMC) entre 25 y 29,9 $\mathrm{kg} / \mathrm{m} 2$ para sobrepeso y sobre $30 \mathrm{~kg} / \mathrm{m} 2$ para obesidad. ${ }^{5}$ Diversos estudios han demostrado que el riesgo de desarrollar HTA es mayor en aquellas personas con sobrepeso u obesidad, así como también se ha evaluado el impacto beneficioso de la reducción del peso en la PA. En el estudio de Framingham se evidenció que la prevalencia de HTA en obesos es el doble que en sujetos con peso normal y un aumento en el peso relativo del $10 \%$ predijo un incremento de $7 \mathrm{mmHg}$ en la PA. ${ }^{2}$ Por otra parte, la fase I del estudio de intervención Trials Of Hypertension Prevention (TOHP) estudió un grupo de 564 sujetos normotensos durante 18 meses, al observar la reducción de la PA en el grupo intervenido para bajar de peso $(n=308)$, los autores determinaron que por cada $\mathrm{kg}$ de peso perdido, la PA sistólica/diastólica disminuye en cifras del orden de 0,43/0,33 mmHg. ${ }^{6}$ La fase II del ensayo estudió a 2.382 sujetos normotensos, con IMC de un $10-60 \%$ por sobre su valor normal, randomizados para recibir consejería sobre reducir el peso y/o disminución del consumo de sodio. Luego de 36 meses de seguimiento, el grupo que bajó de peso disminuyó sus cifras tensionales en $3,7 / 2,7 \mathrm{mmHg}$, el grupo que disminuyó el consumo de sodio bajó su PA en 2,9/1,6 mmHg y el grupo que recibió ambas intervenciones bajó la PA en 4,0/2,8 $\mathrm{mmHg}^{7}{ }^{7}$ Los autores, además, plantean que las intervenciones dirigidas a lograr una baja de peso, producen beneficios que perduran por mucho tiempo después de la intervención activa.

Cabe destacar que la obesidad de distribución abdominal, expresada en términos clínicos como aquellas que se presenta con un perímetro de cintura mayor a $90 \mathrm{~cm}$ en hombres y 80 en mujeres, es el parámetro que mejor se correlaciona con la PA. Esto se debe a que el perímetro abdominal se correlaciona directamente con el tejido adiposo visceral, y la actividad del adipocito visceral constituye el factor metabólicamente activo en la generación del llamado Síndrome Metabólico, caracterizado por inflamación crónica de bajo grado, resistencia a insulina y dislipidemia aterogénica, además de HTA. ${ }^{8}$ Como es de esperar, una disminución en el volumen del tejido adiposo visceral, lo cual se logra hasta con pérdidas de peso del orden de un 5-10\%, conlleva un beneficio metabólico para el paciente hipertenso.

La obesidad condiciona elevación de la PA por diversos mecanismos, que incluyen un aumento de insulina plasmática, aumento de leptina y una activación del sistema nervioso simpático. En relación al aumento de la insulina plasmática, ${ }^{9}$ los mecanismos propuestos mediante los cuales la resistencia a insulina podría aumentar la PA se detallan en la Tabla 1. Con respecto a la leptina, una hormona secretada por el tejido adiposo que se une a su receptor ubicado en el hipotálamo, se postula que alteraría la secreción de óxido nítrico y favorecería la retención de sodio y agua a nivel renal. ${ }^{10}$ Finalmente, en relación a la

\begin{tabular}{l} 
Tabla 1: Efecto de la resistencia a la insulina e \\
hiperinsulinemia sobre la PA. \\
\hline Efectos de la Insulina \\
Aumenta actividad del sistema simpático \\
Aumenta la sensibilidad al sodio \\
Aumenta reabsorción renal de sodio y agua \\
Favorece la entrada de calcio a la célula \\
Estimula factores de crecimiento de células musculares lisas vasculares \\
Favorece la generación de prostaglandinas vasodilatadoras \\
Altera los mecanismos de vasodilatación
\end{tabular}

activación del sistema nervioso simpático, la liberación de catecolaminas y consecuente activación del sistema renina-angiotensina-aldosterona generan retención de sodio, aumentando el gasto cardíaco, lo que aumenta la PA en el contexto de una disminución de los mecanismos vasodilatadores periféricos. ${ }^{11}$ En el corazón, la estimulación simpática se relaciona con hipertrofia e incremento de la contractilidad miocárdica, aumentando el cansancio y los requerimientos de oxígeno. ${ }^{12}$

Actualmente, contamos con tres grandes pilares terapéuticos para el control del peso: cambios de estilo de vida (dieta y ejercicio), tratamiento farmacológico y cirugía bariátrica, siendo la combinación de éstos lo indicado para muchos pacientes. Se ha demostrado que la magnitud de la reducción de la PA varía según el método que se utilice para perder peso. Un estudió constató que un grupo de pacientes que logró bajar $4 \mathrm{~kg}$ de peso mediante dieta, disminuyó la PA sistólica/diastólica en 6,3/3,4 mmHg, mientras que el grupo de pacientes que disminuyó $4 \mathrm{~kg}$ mediante el uso de Orlistat disminuyó la PA sólo en 2,5/2,0 mmHg. ${ }^{13}$ En relación a la cirugía bariátrica, se ha demostrado que la baja de peso se acompaña de una disminución importante de la PA, manteniéndose este efecto mientras se mantenga la pérdida del exceso de peso en el tiempo. ${ }^{14}$ 


\section{Dieta DASH}

Un importante trabajo que aborda la visión multifactorial de la alimentación en la HTA es el estudio Dietary Aproach to Stop Hypertension (DASH), desarrollado en Estados Unidos. ${ }^{15}$ En dicho trabajo se estudió a 459 individuos con y sin HTA, quienes luego de recibir durante 3 semanas una dieta control clásica americana (baja en frutas, verduras y con alto contenido de grasa), fueron randomizados para recibir dos tipos de dieta: una dieta con alto aporte de frutas y verduras o la dieta DASH que, además de frutas y verduras, aportaba alto contenido de productos lácteos descremados. Luego de 8 semanas de seguimiento, el grupo con la dieta DASH fue el que presentó el mayor beneficio en la PA, reduciendo 5,5/3,0 mmHg más que la dieta control típica americana y 2,7/1,9 $\mathrm{mmHg}$ que la dieta que sólo aportó frutas y verduras. En aquellos pacientes hipertensos ( $\mathrm{n}=133)$, los resultados fueron aún más pronunciados al disminuir las cifras de PAen
$11,4 / 5,5 \mathrm{mmHg}$ ya a las 2 semanas de intervención, siendo este efecto antihipertensivo comparable al tratamiento farmacológico con monoterapia en HTA leve ${ }^{15}$. Además, en pacientes sin HTA, las cifras de PA también disminuyeron, dando especial relevancia a la dieta DASH para la prevención primaria de esta enfermedad.

En un segundo trabajo llamado "DASH sodio" se realizó un seguimiento de 412 adultos randomizados para seguir la dieta control típica americana o la dieta DASH, junto con estratificar el consumo de sodio en 3 grupos $(50,100 \mathrm{y}$ $150 \mathrm{mmol} /$ día equivalentes a 1,$15 ; 2,3$ y 3,4 gr/día). Luego de 30 días, la reducción del contenido de sodio disminuyó la PA en ambas dietas, pero a cualquier nivel de aporte de sodio el mejor beneficio se obtuvo en el grupo de la dieta DASH. Como era de esperar, el mejor resultado en el descenso de la PA se observó en el grupo de pacientes con la dieta DASH además de la restricción máxima de sodio de $50 \mathrm{mmol} /$ día. $^{16}$

\begin{tabular}{|c|c|c|}
\hline Alimento & Porción recomendada & Efecto beneficioso \\
\hline Granos y productos con granos enteros & $\begin{array}{l}\text { 7-8 / día } \\
\text { Porción: } 1 \text { rebanada de pan integral, } 1 / 2 \\
\text { taza de pasta o cereal. }\end{array}$ & $\begin{array}{l}\text { Efecto beneficioso } \\
\text { Fuente mayor de energía y fibra }\end{array}$ \\
\hline Vegetales & $\begin{array}{l}\text { 4-5 / día } \\
\text { Porción: } 1 \text { taza de vegetales crudos, } 1 / 2 \\
\text { taza de vegetales cocidos. }\end{array}$ & Fuente rica en potasio, magnesio y fibra \\
\hline Fruta & $\begin{array}{l}\text { 4-5 / día } \\
\text { Porción: } 1 \text { fruta mediana, } 1 / 2 \text { taza de fruta } \\
\text { picada. }\end{array}$ & $\begin{array}{l}\text { Fuente importante de potasio, magnesio } \\
\text { y fibra }\end{array}$ \\
\hline Lácteos descremados o semi-descremados & $\begin{array}{l}2-3 \\
\text { Porción: Un vaso de } 200 \mathrm{ml} \text { de leche, } 1 \\
\text { taza de yogurt. }\end{array}$ & Fuente mayor de calcio y proteínas \\
\hline Carne y pescado & $\begin{array}{l}2 \text { o menos /día } \\
\text { Porción: } 85 \text { gr de carne o pescado } \\
\text { cocinada. }\end{array}$ & Fuente rica en proteína y magnesio \\
\hline Semillas, legumbres y frutos secos & $\begin{array}{l}4-5 / \text { semana } \\
\text { Porción: } 1 / 3 \text { taza de frutos secos, } 2 \\
\text { cucharadas de semillas, } 1 / 2 \text { taza de legum- } \\
\text { bres cocinadas. }\end{array}$ & $\begin{array}{l}\text { Fuente rica en energía, magnesio, potasio, } \\
\text { proteína y fibra }\end{array}$ \\
\hline Aceite y grasa & $\begin{array}{l}\text { 2-3 / día } \\
\text { Porción: } 1 \text { cucharadita de margarina } \\
\text { light, } 1 \text { cucharada de mayonesa light, } 1 \\
\text { cucharadita de aceite vegetal. }\end{array}$ & $\begin{array}{l}\text { Fuente de energía y ácidos grasos } \\
\text { esenciales }\end{array}$ \\
\hline Azúcar & $\begin{array}{l}\text { Porción: } 1 \text { cucharadita de azúcar, } 1 \\
\text { cucharadita de miel o mermelada }\end{array}$ & Fuente de energía \\
\hline \multicolumn{3}{|c|}{$\begin{array}{l}\text { Estas recomendaciones están basadas en una dieta de } 2.000 \text { kcal/día, por lo que la recomendación en el número de porcio- } \\
\text { nes debiera adecuarse a los requerimientos energéticos de cada paciente. }\end{array}$} \\
\hline
\end{tabular}


Cabe destacar que el beneficio de la dieta DASH no es explicable por la reducción de peso, consumo de sodio o de alcohol. Muy por el contrario, se debe a diversos componentes que actúan de manera independiente, destacando el efecto diurético que involucra la excreción de sodio, un efecto antioxidante directo de los polifenoles sobre la inflamación vascular y el estrés oxidativo, un efecto sobre la relajación del lecho vascular y una reducción de marcadores inflamatorios como proteína C-reactiva mediante agentes fitoquímicos, entre otros. ${ }^{15-16}$

En la Tabla 2 se describen los detalles de la Dieta Dash.

\section{Actividad Física}

La actividad física regular se asocia a menor riesgo de enfermedad cardiovascular, mortalidad cardiovascular y mortalidad total. Sus efectos benéficos se han atribuido principalmente a la disminución del porcentaje de grasa corporal, de la resistencia a la insulina, al aumento del colesterol HDL, mejoría de la hipertensión y de la función endotelial, junto con una disminución de los niveles de factores inflamatorios, como la proteína C-reactiva ultrasensible. ${ }^{17} \mathrm{El}$ efecto antihipertensivo parece ser producto de una disminución de la actividad adrenérgica y aumento de sustancias vasodilatadoras como prostaglandinas y óxido nítrico18. Este efecto parece ser independiente del beneficio sobre la PA debido a la baja de peso que se logra mediante la actividad física. ${ }^{19}$

Los resultados de una revisión sistemática con 105 estudios randomizados indican que el ejercicio logra disminuir la PA en 5,0/3,0 mmHg. ${ }^{19}$ Este efecto parece ser dosis-dependiente hasta cierto nivel de actividad, sobre el cual no hay un mayor efecto en la PA. Así lo demostró un estudio con 207 individuos hipertensos randomizados a realizar actividad física en 5 rangos de intensidad (minutos/semana). Luego de 8 semanas las personas que realizaban $61-90 \mathrm{~min} / \mathrm{semana}$ presentaron un mayor beneficio que aquellas que realizaban 31-60 $\mathrm{min} / \mathrm{semana}$, pero no existió un beneficio adicional en aquellos que realizaban sobre los $91 \mathrm{~min} /$ semana. Por lo tanto, no es necesario realizar ejercicio de manera agresiva para alcanzar la mayor reducción en las cifras tensionales, pero éste debe ser mantenido a lo largo del tiempo, ya que aparentemente el grado de acondicionamiento físico (la respuesta fisiológica frente a la actividad física regular, medido en base al tiempo que un individuo es capaz de realizar un ejercicio intenso en ausencia de ciertos síntomas) estaría directamente asociado al beneficio antihipertensivo. En relación a esto, en el estudio Coronary Artery Risk Development in Young Adults (CARDIA) con 4.618 adultos, tanto el acondicionamiento como la actividad física, demostraron estar inversamente asociadas con la incidencia de HTA a 20 años de seguimiento. Además de constatar un efecto más marcado en el grupo con mayor acondicionamiento físico, los autores señalan que un 34\% de la incidencia de HTA podría haber sido evitada si los individuos hubieran pasado a una categoría de acondicionamiento mayor. ${ }^{21}$

\section{Minerales y Otros Nutrientes}

Se han reportado asociaciones significativas entre la PA y la ingesta de potasio, magnesio, calcio, fibra y proteínas. Sin embargo, en ensayos clínicos el efecto en la PA tras la ingesta de dichos nutrientes ha sido inconsistente y controversial. ${ }^{19} \mathrm{Al}$ parecer, el efecto de la administración de cada nutriente por si sólo sería muy discreto como para permitir su detección en los ensayos clínicos. No obstante, la sumatoria de estos componentes tendría un efecto antihipertensivo tangible. La evidencia más convincente es la disponible para el potasio.

Potasio: Diversos estudios han evidenciado que la suplementación con potasio reduce la PA en sujetos normotensos e hipertensos. Un meta-análisis de 27 estudios demostró que un aumento de $44 \mathrm{mmol} /$ día (equivalentes a 1,72 $\mathrm{gr} /$ día) de potasio da cuenta de una disminución de 3,5/2,5 mmHg en sujetos hipertensos y de $0,9 / 0,3 \mathrm{mmHg}$ en sujetos normotensos. ${ }^{22}$ Según otro meta-análisis de 41 estudios controlados, el mayor efecto del consumo de potasio se observa en las personas que presentan una mayor sensibilidad al sodio y un mayor consumo del mismo. Esto se debe a que el mayor aporte de potasio ejerce un poderoso efecto inhibitorio dosis dependiente de la sensibilidad al sodio (presente en un 26\% de los normotensos y en 51\% de los hipertensos), es decir, inhibe el aumento de la PA en respuesta a una mayor ingesta de cloruro de sodio. ${ }^{23}$ Otros mecanismos corresponden a un efecto modulador de barorreceptores, menor efecto vasocontrictor de noradrenalina y angiotensina II, estimulación de calicreína renal (efecto natriurético), entre otros. ${ }^{22,23}$

Basándose en estos estudios, la recomendación dietaria de potasio corresponde a $90 \mathrm{mmol} /$ día (aproximadamente 3.5 gr al día), siendo preferible obtener el potasio mediante una dieta rica en verduras y frutas más que a través de 
un suplemento alimentario. ${ }^{24}$

Magnesio: Estudios en animales han demostrado que la deficiencia de magnesio se asocia a un aumento de la PA y su suplementación disminuye el riesgo de desarrollar HTA. ${ }^{25}$ Sin embargo, los resultados que asocian ingesta de magnesio con PA han sido poco concluyentes en humanos. En la cohorte de Framingham no hubo asociación entre el magnesio sérico y la incidencia de HTA. ${ }^{26}$ Aun así, en un meta-análisis de 20 ensayos clínicos, la suplementación con magnesio resultó en una leve pero significativa reducción promedio de la PA de $0,6 / 0,8 \mathrm{mmHg}^{27}$ Este efecto puede atribuirse principalmente al efecto vasodilatador del magnesio.

\section{Vitamina D}

La vitamina D o 25-hidroxi-colecalciferol ha sido recientemente vinculada a una amplia gama de condiciones clínicas incluyendo la HTA. Esto se explica porque el receptor de vitamina $\mathrm{D}$ se encuentra ampliamente presente en el organismo, incluyendo tejidos como el endotelio, la célula muscular lisa y los cardiomiocitos. Diversos estudios epidemiológicos han constatado una asociación inversa entre niveles de PA y el tiempo promedio de exposición a la radiación solar, principal fuente de obtención de vitamina D. ${ }^{28}$ Apoyando esta observación, se ha demostrado que existe una clara variabilidad estacional en las cifras de PA, siendo ésta significativamente más elevada en invierno y más baja en los meses de verano. ${ }^{28}$ El estudio NHANES II estudió a 3.577 adolecentes y demostró que aquellas con niveles plasmáticos bajos de vitamina $\mathrm{D}$ presentaron el doble de probabilidad (OR 2,36; $95 \%$ IC) de desarrollar HTA, en comparación a adolecentes con niveles plasmáticos más altos de vitamina D. ${ }^{29}$ Un estudio de intervención demostró una disminución significativa de la PA sistólica del orden del 9,3\% mediante la suplementación con 800 UI/día de vitamina D y $1.200 \mathrm{mg} /$ día de calcio por 8 semanas, comparado con la suplementación aislada de calcio. ${ }^{30}$ Otro reciente estudio de intervención con 283 pacientes afroamericanos, quienes fueron randomizador para recibir suplementación con vitamina $\mathrm{D}$ en dosis de 1.000 , 2.000 o $4.000 \mathrm{U}$ al día. Luego de tres meses, se observó disminución en la PA sistólica del orden de $-0,66 \mathrm{mmHg}$, $-3,5 \mathrm{mmHg}$ y $-4,0 \mathrm{mmHg}$ en los grupos que recibieron $1.000,2.000$ y $4.000 \mathrm{U} /$ día respectivamente. No se obser- vó efecto en la PA diastólica. Los autores concluyeron que por cada 1.000 U/día del suplementación con vitamina D se logra una disminución de $1,4 \mathrm{mmHg}$ en la PA sistólica $(\mathrm{p}=0,04)$. $^{31}$

Se han propuesto distintos mecanismos para explicar el rol de la vitamina D en la PA. En primer lugar, se ha descrito que la vitamina $\mathrm{D}$ favorece la actividad del factor natriurético auricular mediante una amplificación de la expresión de su receptor. ${ }^{32}$ En segundo lugar, el hiperparatiroidismo secundario que genera el déficit de vitamina D conlleva efectos desfavorables como hipertrofia ventricular izquierda y fibrosis, tanto miocárdica como del lecho vascular, facilitando así el desarrollo de HTA. En tercer lugar, se ha descrito el efecto de la vitamina $\mathrm{D}$ en la regulación negativa del sistema renina-angiotensina. ${ }^{33}$ Por último, se ha propuesto también un mecanismo relacionada con la resistencia a la insulina, ya que bajas concentraciones de vitamina $\mathrm{D}$ se relacionan con intolerancia a la glucosa y resistencia a la insulina. ${ }^{34}$

No se ha establecido una relación causal entre el déficit de vitamina D y la HTA. La información disponible en la actualidad, basada en estudios clínicos controlados, es insuficiente para definir la efectividad de la suplementación con vitamina D para la prevención o tratamiento de la HTA.

\section{Ácidos grasos Omega-3}

Se ha demostrado una relación significativa entre el consumo de grasas saturadas y la PA, lo cual se ha corroborado en estudios de animales que han mostrado un progresivo aumento de su PA con dietas altas en grasas saturadas. ${ }^{35}$ Esto estaría relacionado con el aumento del tejido adiposo visceral y la resistencia a la insulina producto de la alta ingesta calórica. ${ }^{36}$

Por el contrario, la ingesta de ácidos grasos poli insaturados del tipo omega-3 (principalmente el eicosapentaenoico o EPA y el decosahexaenoico o DHA), componente importante de la dieta mediterránea, ha demostrado reducir las cifras de PA en tanto en hipertensos ya establecidos como en sujetos sanos. 37

En el meta-análisis de Morris y colaboradores, quienes evaluaron 31 estudios controlados sobre la suplementación con omega-3 en hipertensos y normotensos, los autores concluyeron que la ingesta promedio de 4,8 gr/día de ácido graso omega-3 de origen marino reduce la PA 
de manera estadísticamente significativa, en valores del orden de 3,0/1,5 mmHg. Esta disminución de las cifras tensionales fue más acentuada en aquellos sujetos hipertensos, mostrando una creciente intensidad dosis dependiente a partir del aporte de $3 \mathrm{gr} /$ día de omega- $3 .{ }^{37} \mathrm{Se}$ cree que existe un mayor efecto antihipertensivo asociado al consumo de DHA que aquel observado mediante el consumo de EPA.

Los ácidos grasos omega-3 ejercen su efecto hipotensor mediante la producción de eicosanoides con actividad vasodilatadora, como la prostaglandina $\mathrm{E} 3$, prostaciclina I3 y el tromboxano A3, y disminuyendo la producción de eicosanoides con carácter vasocontrictor como el tromboxano A2, además del efecto estimulador de la síntesis de óxido nítrico por parte del lecho vascular. ${ }^{38}$

\section{Flavonoides}

Los flavonoides son compuestos polifenólicos presentes en altas concentraciones en frutas, verduras, granos, legumbres, té, cacao, cerveza y vino. Existe un gran número de flavonoides, tales como flavonoles, taninos, antocianinos, flavonas, isoflavonas y flavononas, entre otros, a los que se les ha atribuido diversas propiedades, lo que refleja su notable variedad química. Estudios epidemiológicos llevados a cabo entre 1958 y 1970, han demostrado una asociación inversa entra la ingesta diaria de flavonoides y la mortalidad cardiovascular, ${ }^{39}$ lo cual podría explicarse por su combinación de propiedades antioxidantes, antiagregantes plaquetarios y vasodilatadores.

Algunos estudios experimentales en HTA sugieren que el extracto de algunos flavonoides tendría un efecto antihipertensivo al aumentar la producción de óxido nítrico endotelial. ${ }^{40}$ Se ha reportado que la quercetina (flavonol altamente presente en frutas y verduras) ejerce efectos antihipertensivos y reduce la hipertrofia del ventrículo izquierdo, la disfunción endotelial y mejora el estatus oxidativo hepático en ratas hipertensas. ${ }^{41}$ Otro estudio evaluó el efecto de la administración de té de Hibiscus (Hibiscus sabdariffa) en la HTA esencial. Para este caso, se seleccionaron al azar pacientes con HTA moderada, encontrando una reducción del 11,2\% de las cifras de PA sistólica, así como un decremento del $10,7 \%$ de la PA diastólica a los 15 días después de iniciado el protocolo. ${ }^{42}$

Un reciente análisis de 31 estudios controlados y randomizados evaluó el efecto antihipertensivo de la epicatequi- na, un tipo de flavonoide presente en el cacao. Se demostró un efecto estadísticamente significativo en la reducción de la PA sistólica y diastólica, con cifras promedio del orden de 4,1/2,0 mmHg mediante la ingesta de $25 \mathrm{mg} /$ día de epicatequina. ${ }^{43} \mathrm{Se}$ cree que uno de sus principales efectos es la elevación de los niveles de óxido nítrico en la sangre y su consecuente efecto vasodilatador.

\section{Sacarosa y Fructosa}

Se ha demostrado una asociación entre el consumo de sacarosa y fructosa con un aumento de las cifras de PA. Un estudio transversal que usó la base de datos del NHANES con 4.528 pacientes sin diagnóstico de HTA, demostró que aquellos individuos cuyo consumo de fructosa superaba el valor promedio ( $74 \mathrm{gr} /$ día de fructosa, provenientes de bebidas azucaradas), mostraban un riesgo del orden del $77 \%$ de presentar PA por sobre 160/100 mmHg, ajustado por variables demográficas, nivel de actividad física, ingesta de calorías totales, ingesta de carbohidratos, sal y vitamina $\mathrm{C}^{44}$

Otro estudio con 810 pacientes demostró que al dejar de consumir 1 bebida azucarada al día, se producía una disminución significativa de la PA de 2,0/1,2 mmHg, ajustado por variaciones en el IMC. ${ }^{45}$

Los mecanismos biológicos por los cuales el consumo de sacarosa y fructosa elevan la PA son la inhibición de la enzima óxido nítrico sintetasa a nivel endotelial, estimulación del sistema nervioso simpático, aumento de la absorción intestinal de sodio y aumento del ácido úrico plasmático, este último es mediado por un aumento de la actividad del sistema renina- angiotensina-aldosterona y una hipertensión glomerular. ${ }^{46}$

\section{Cafeína}

La cafeína ejerce una variedad de acciones farmacológicas a nivel central y periférico, cuyo principal mecanismo de acción es el antagonismo de la adenosina. La adenosina es un potente neuromodulador endógeno que presenta acciones reguladoras en los sistemas cardiovascular y nervioso central. Mediante este mecanismo, la cafeína produce una vasoconstricción del lecho vascular e incrementa la PA de manera aguda en cifras del orden de $5-15 \mathrm{mmHg}{ }^{47}$ Aunque estos hallazgos indican que la cafeína podría contribuir al desarrollo de HTA, los estudios actuales al respecto son controversiales, ya que el efecto de la cafeína 
sobre la PA es proporcional a sus niveles sistémicos luego de ser consumida, por lo que es un efecto pasajero, y a largo plazo ejerce un efecto mínimo. ${ }^{48}$ Cabe destacar que individuos que consumen cafeína de manera regular presentan menos variación de su PA frente a la ingesta aguda de cafeína, sugiriendo cierta tolerancia al efecto adrenérgico inducido por esta sustancia. ${ }^{49}$

\section{Alcohol}

Se ha documentado un efecto protector del consumo moderado de alcohol en la enfermedad cardiovascular, observándose el máximo beneficio en los consumidores de vino. Esto significa que con una dosis óptima de consumo (alrededor de 20 gr de etanol al día) se obtiene un menor riesgo de mortalidad cardiovascular que los abstemios y los consumidores excesivos (curva en " $\mathrm{J}$ "). ${ }^{50}$

El efecto del alcohol en la PA se produce al sobrepasar un cierto umbral de consumo crónico de alrededor de 2-3 bebidas al día (20 a $34 \mathrm{Gr}$ de alcohol por día) de cualquier tipo de alcohol, mientras que un consumo por debajo de 20 gr/día parece no tener efecto en la PA. Esta relación ha sido constatada en diversos estudios epidemiológicos y parece afectar por igual a ambos sexos y todas las edades. ${ }^{51}$

Así, a 4 años de seguimiento, el "estudio de las enfermeras" ( $\mathrm{n}=58.218)$ mostró un mayor riesgo de desarrollar HTA de 1,4 veces en las mujeres que consumía de 20 a 34 $\mathrm{g}$ de alcohol y de 1,9 veces en las que consumía sobre 35 gr/día, en comparación con el grupo de las abstemias. ${ }^{51}$

Los mecanismos propuestos por los cuales el consumo de alcohol eleva la PA incluyen un efecto directo sobre la resistencia vascular, aumento de la actividad simpática, disminución del magnesio intracelular, disminución de la producción de óxido nítrico y a largo plazo favorece la resistencia a la insulina e intolerancia a la glucosa, ambas con el efecto presor ya descrito. ${ }^{52}$

\section{Conclusión}

La evidencia expuesta tiene trascendencia para la salud pública en Chile. La HTA es una enfermedad altamente prevalente en nuestro país. La reciente Encuesta Nacional de Salud (ENS) mostró una prevalencia de HTA nacional del $26,9 \%$, un $65 \%$ de conocimiento de la enfermedad, sólo un 37,6\% en tratamiento y un control satisfactorio en apenas un $16,5 \%{ }^{5}$ Analizando la ENS más en detalle, nos encontramos con un escenario en el cual predomina un estilo de vida poco saludable y múltiples factores nutricionales que facilitan el desarrollo de HTA en nuestra población, tales como el elevado nivel de obesidad y sobrepeso, la falta de actividad física, la ingesta excesiva de sodio, el consumo de alcohol, el tabaquismo, la deficiente ingesta de frutas y verduras, de potasio y de pescado. ${ }^{5}$

Todo lo anterior, explica la epidemia nacional de HTA y nos permite reflexionar sobre la importancia de dar a conocer las medidas dietéticas que hemos presentado, lo cual nos permitirá un mejor control de la PA en pacientes hipertensos y un retraso de la aparición de esta enfermedad en sujetos sanos, con un impacto favorable en la expectativa de vida.

El estudio PREDIMED (Prevención con Dieta Mediterránea) muestran cómo la incorporación de un patrón de alimentación saludable a nivel poblacional permite disminuir los niveles de PA de manera significativa. ${ }^{53}$ Es por esto que nosotros planteamos que algunas medidas globales, tales como la reducción de sodio en alimentos industriales, la mediterranización de la dieta, el etiquetado adecuado de los alimentos y la inversión en campañas de educación nutricional, (aun cuando éstas precisan de un gran esfuerzo por parte de las autoridades sanitarias), serán necesarias para lograr un verdadero cambio en la dieta de nuestra población y así, en la prevalencia de HTA en ella. 


\section{Referencias:}

1. LEWINGTON S, CLARKE R, QIZILBASH N, PETO R, COLLINS R Prospective Studies Collaboration. Age-specific relevance of usual blood pressure to vascular mortality: a meta-analysis of individual data for one million adults in 61 prospective studies. Lancet. 2002; 360: 1903-1913.

2. GORDON T, KANNEL WB. Predisposition to atherosclerosis in the head, heart, and legs. The Framingham study. JAMA. 1972; 221:661-666.

3. APPEL LJ. Lifestyle modification as a means to prevent and treat high blood pressure. J Am Soc Nephrol. 2003;14: S99-S102.

4. MIDGLEY JP, MATTHEW AG, GREENWOOD CM, LOGAN AG. Effect of reduced dietarysodium on blood pressure: a meta-analysis of randomized controlled trials. JAMA. 1996; 275: 1590-1597.

5. MINSAL. Encuesta Nacional de Salud 2010. Disponible en http://www.redsalud.gov.cl/portal/url/item/99bbf09a9 08d3eb8e04001011f014b49.pdf (Consultado el 6 de Noviembre de 2012).

6. STEVENS VJ, CORRIGAN SA, OBARZANEK E, BERNAUER E, COOK NR, HEBERT P, et al. Weight loss intervention in phase 1 of the Trials of Hypertension Prevention. The TOHP Collaborative Research Group. Arch Intern Med. 1993; 153: 849-858.

7. Effects of weight loss and sodium reduction intervention on blood pressure and hypertension incidence in overweight people with high-normal blood pressure. The Trials of Hypertension Prevention, phase II. The Trials of Hypertension Prevention Collaborative Research Group. Arch Intern Med. 1997; 157: 657-667.

8. ECKEL R, GRUNDY S, ZIMMET P. The metabolic syndrome. Lancet. 2005;365:1415-28.

9. LANDSBERG L. Insulin-mediated sympathetic stimulation: role in the pathogenesis of obesity-related hypertension (or, how insulin affects blood pressure, and why). J
Hypertens. 2001; 19: 523-528.

10. VILLARREAL D, REAMS G, SAMAR H, SPEAR R, FREEMAN RH. Effects of chronic nitric oxide inhibition on the renal excretory response to leptin. Obes Res. 2004; 12 : 1006-1010.

11. SARZANI R, SALVI F, DESSİ-FULGHERI P, RAPPELLI A. Renin-angiotensin system, natriuretic peptides, obesity, metabolic syndrome, and hypertension: an integrated view in humans. J Hypertens. 2008; 26: 831-843.

12. NAKAYAMA Y, MIYANO H, SHISHIDO T, INAGAKI M, KAWADA T, SUGIMACHI M, et al. Laterality in direct and indirect inotropic effects of sympathetic stimulation in isolated canine heart. Jpn J Physiol. 2001; 51: 365-370

13. HORVATH K, JEITLER K, SIERING U, STICH AK, SKIPKA G, GRATZER TW, et al. Long-term effects of weight-reducing interventions in hypertensive patients: systematic review and meta-analysis. Arch Intern Med. 2008; 168: 571-580.

14. SJÖSTRÖM L, LINDROOS AK, PELTONEN M, TORGERSON J, BOUCHARD C, CARLSSON B, et al. Lifestyle, diabetes, and cardiovascular risk factors 10 years after bariatric surgery. N Engl J Med. 2004; 351: 2683-2693.

15. APPEL LJ, MOORE TJ, OBARZANEK E, VOLLMER WM, SVETKEY LP, SACKS FM, et al. A clinical trial of the effects of dietary patterns on blood pressure. DASH Collaborative Research Group. N Engl J Med. 1997; 336: 1117-1124.

16. VOLLMER WM, SACKS FM, ARD J, APPEL LJ, BRAY GA, SIMONS-MORTON DG, et al. Effects of diet and sodium intake on blood pressure: subgroup analysis of the DASH-sodium trial. Ann Intern Med. 2001; 135: 10191028.

17. ARONSON D, SHEIKH-AHMAD M, AVIZOHAR O, KERNER A, SELLA R, BARTHA P, et al. C-Reactive protein is inversely related to physical fitness in middle-aged subjects. Atherosclerosis. 2004; 176: 173-179 
18. GOTO C, HIGASHI Y, KIMURA M, NOMA K, HARA $\mathrm{K}$, NAKAGAWA K, et al. Effect of different intensities of exercise on endothelium-dependent asodilation in humans: role of endothelium-dependent nitric oxide and oxidative stress. Circulation. 2003; 108: 530-535.

19. DICKINSON HO, MASON JM, NICOLSON DJ, CAMPBELL F, BEYER FR, COOK JV, et al. Lifestyle interventions to reduce raised blood pressure: a systematic review of randomized controlled trials. J Hypertens. 2006; 24: 215-233.

20. ISHIKAWA-TAKATA $\mathrm{K}$, OHTA T, TANAKA H. How much exercise is required to reduce blood pressure in essential hypertensives: a dose-response study. Am J Hypertens. 2003; 16: 629-633.

21. CARNETHON MR, EVANS NS, CHURCH TS, LEWIS CE, SCHREINER PJ, JACOBS DR JR, et al . Joint associations of physical activity and aerobic fitness on the development of incident hypertension: coronary artery risk development in young adults. Hypertension. 2010; 56: 49-55.

22. GELEIJNSE JM, KOK FJ, GROBBEE DE. Blood pressure response to changes in sodium and potassium intake: a metaregression analysis of randomised trials. J Hum Hypertens. 2003; 17: 471-480.

23. MORRIS RC JR, SEBASTIAN A, FORMAN A, TANAKA M, SCHMIDLIN O. Normotensive salt sensitivity: effects of race and dietary potassium. Hypertension. 1999; 33: 18-23.

24. WHELTON PK, HE J, APPEL LJ, CUTLER JA, HAVAS S, KOTCHEN TA, et al. Primary prevention of hypertension: clinical and public health advisory from The National High Blood Pressure Education Program. JAMA. 2002; 288: 1882-1888.

25. KH R, KHULLAR M, KASHYAP M, PANDHI P, UPPAL R. Effect of oral magnesium supplementation on blood pressure, platelet aggregation and calcium handling in deoxycorticosterone acetate induced hypertension in rats. J Hypertens. 2000; 18: 919-926.
26. KHAN AM, SULLIVAN L, MCCABE E, LEVY D, VASAN RS, WANG TJ. Lack of association between serum magnesium and the risks of hypertension and cardiovascular disease. Am Heart J. 2010; 160: 715-720.

27. JEE SH, MILLER ER 3RD, GUALLAR E, SINGH VK, APPEL LJ, KLAG MJ. The effect of magnesium supplementation on blood pressure: a meta-analysis of randomized clinical trials. Am J Hypertens. 2002; 15: 691-696.

28. WOODHOUSE PR, KHAW KT, PLUMMER M. Seasonal variation of blood pressure and its relationship to ambient temperature in an elderly population. J Hypertens. 1993; 11: $1267-1274$.

29. REIS JP, VON MÜHLEN D, MILLER ER 3RD, MICHOS ED, APPEL LJ. Vitamin D status and cardiometabolic risk factors in the United States adolescent population. Pediatrics. 2009; 124: e371-e379.

30. PFEIFER M, BEGEROW B, MINNE HW, NACHTIGALL D, HANSEN C. Effects of a short-term vitamin $\mathrm{D}$ (3) and calcium supplementation on blood pressure and parathyroid hormone levels in elderly women. J Clin Endocrinol Metab. 2001; 86: 1633-1637.

31. FORMAN JP, SCOTT JB, NG K, DRAKE BF, SUAREZ EG, HAYDEN DL, et al. Effect of vitamin d supplementation on blood pressure in blacks. Hypertension. 2013; 61: $779-85$.

32. CHEN S, NI XP, HUMPHREYS MH, GARDNER DG. 1,25 dihydroxyvitamin $\mathrm{d}$ amplifies type a natriuretic peptide receptor expression and activity in target cells. J Am Soc Nephrol. 2005; 16: 329-339.

33. LI YC, KONG J, WEI M, CHEN ZF, LIU SQ, CAO LP. 1,25 -Dihydroxyvitamin $\mathrm{D}(3)$ is a negative endocrine regulator of the renin-angiotensin system. J Clin Invest. 2002; 110: $229-238$

34. MITRI J, MURARU MD, PITTAS AG. Vitamin D and type 2 diabetes: a systematic review. Eur J Clin Nutr. 2011; 65: 1005-15. 
35. TAMAYA-MORI N, UEMURIA K, IGUCHI A. Gender differences in the dietary lard-induced increase in blood pressure in rats. Hypertension 2002; 39: 1015-20.

36. UEMURA K, MORI N. Influence of age and sex on highfat diet-induced increase in blood pressure. Nagoya J Med Sci. 2006; 68: 109-14.

37. MORRIS MC, SACKS F, ROSNER B. Does fish oil lower blood pressure? A meta-analysis of controlled trials. Circulation. 1993; 88: 523-533.

38. YIN K, CHU ZM, BEILIN LJ. Blood pressure and vascular reactivity changes in spontaneously hypertensive rats fed fish oil. Br J Pharmacol. 1991; 102: 991-997.

39. HERTOG MG, KROMHOUT D, ARAVANIS C, BLACKBURN H, BUZINA R, FIDANZA F, et al. Flavonoid intake and long-term risk of coronary heart disease and cancer in the seven countries study. Arch Intern Med. 1995; 155: 381-386.

40. STEFFEN Y, GRUBER C, SCHEWE T, SIES H. MonoO-methylated flavanols and other flavonoids as inhibitors of endothelial NADPH oxidase. Arch Biochem Biophys. 2008; 469: 209-219.

41. DUARTE J, GALISTEO M, OCETE MA, PÉREZ-VIZCAINO F, ZARZUELO A, TAMARGO J. Effects of chronic quercetin treatment on hepatic oxidative status of spontaneously hypertensive rats. Mol Cell Biochem. 2001; 221: 155-160.

42. HAJI FARAJI M, HAJI TARKHANI A. The effect of sour tea (Hibiscus sabdariffa) on essential hypertension. J Ethnopharmacol. 1999; 65: 231-236.

43. ELLINGER S, REUSCH A, STEHLE P, HELFRICH HP. Epicatechin ingested via cocoa products reduces blood pressure in humans: a nonlinear regression model with a Bayesian approach. Am J Clin Nutr. 2012; 95: 1365-1377.

44. JALAL DI, SMITS G, JOHNSON RJ, CHONCHOL M. Increased fructose associates with elevated blood pressure. J Am Soc Nephrol. 2010; 21: 1543-1949.
45. CHEN L, CABALLERO B, MITCHELL DC, LORIA C, LIN PH, CHAMPAGNE CM, et al. Reducing consumption of sugar-sweetened beverages is associated with reduced blood pressure: a prospective study among United States adults. Circulation. 2010; 121: 2398-2406.

46. JOHNSON RJ, SANCHEZ-LOZADA LG, NAKAGAWA $\mathrm{T}$. The effect of fructose on renal biology and disease. J Am Soc Nephrol. 2010;12: 2036-2039.

47. SMITS P, THIEN T, VAN'T LAAR A. Circulatory effects of coffee in relation to the pharmacokinetics of caffeine. Am J Cardiol. 1985; 56: 958-963.

48. BIRKETT NJ, LOGAN AG. Caffeine-containing beverages and the prevalence of hypertension. J Hypertens Suppl. 1988; 6: S620-S622.

49. CORTI R, BINGGELI C, SUDANO I, SPIEKER L, HÄNSELER E, RUSCHITZKA F, et al. Coffee acutely increases sympathetic nerve activity and blood pressure independently of caffeine content: role of habitual versus nonhabitual drinking. Circulation. 2002; 106: 2935-2940.

50. COSTANZO S, DI CASTELNUOVO A, DONATI MB, IACOVIELLO L, DE GAETANO G. Wine, beer or spirit drinking in relation to fatal and non-fatal cardiovascular events:a meta-analysis. Eur J Epidemiol. 2011; 26: 833850 .

51. WITTEMAN JC, WILLETT WC, STAMPFER MJ, COLDITZ GA, KOK FJ, SACKS FM, et al. Relation of moderate alcohol consumption and risk of systemic hypertension in women. Am J Cardiol. 1990; 65: 633-637.

52. WAKABAYASHI I, HATAKE K. Effects of ethanol on the nervous and vascularsystems: the mechanisms of alcoholinduced hypertension. Nihon Eiseigaku Zasshi. 2001; 55: 607-617.

53. ESTRUCH R, MARTÍNEZ-GONZÁLEZ MA, CORELLA D, SALAS-SALVADÓ J, RUIZ-GUTIÉRREZ V, COVAS MI, et al. Effects of a Mediterranean-style diet on cardiovascular risk factors: a randomized trial. Ann Intern Med. 2006; 145:1-11. 